Pain Physician. 2005;8:297-308, ISSN 1533-3159

\title{
The Pharmacology of Sedation
}

\author{
James D. Colson, MD
}

Sedation is commonly provided for patients undergoing interventional pain management procedures. Although many pain management interventionalists have trained in anesthesiology, the use of sedatives, analgesics and hypnotics, as well as monitoring techniques, may vary considerably between practices.

This review provides an update on re-

The term sedation encompasses a progressive continuum of stages ranging from minimal sedation ("anxiolysis") through moderate sedation/analgesia ("conscious sedation"), deep sedation, and ultimately general anesthesia. Each stage represents a progressive degree of central nervous system (CNS) depression with impaired levels of cognition and consciousness, decreased responsiveness to stimuli, loss of protective reflexes, and diminished respiratory effort and cardiovascular performance. The therapeutic goal of sedation is to provide calm, comfort, pain relief, and cooperation of a patient undergoing a procedure that often evokes anxiety, discomfort, and pain, but which may require full patient cooperation.

Sedation techniques are employed to facilitate diagnostic or therapeutic procedures requiring varying degrees of anxiolysis and/or analgesia, such as magnetic resonance imaging (MRI), endoscopy, setting a bone fracture, or an interventional

From: Department of Anesthesiology, University of Michigan, Ann Arbor, MI

Address Correspondence:

James D. Colson, MS, MD, 1 H2 47 UH, 1500 E.

Medical Center Drive, Ann Arbor, MI 48130

E-mail: colsonj@umich.edu

Disclaimer: There was no external funding in prepa-

ration of this manuscript.

Conflict of Interest: None

Manuscript received on $3 / 12 / 2005$

Revision submitted on 5/14/2005

Accepted for publication on $5 / 15 / 2005$ cent advances in the pharmacology of drugs commonly used for sedation, anxiolysis and analgesia. The information should also be useful for non-anesthesiologists who provide sedation for their patients. In addition to providing the right drug for the procedure, patient monitoring must be appropriate for the patient and the clinical circumstances. Undertaking a sedation analgesic course of therapy in support of an interventional procedure will require that patients are monitored appropriately during the course of treatment. Guidelines for monitoring patients during sedation are available.

Keywords: sedation, anxiolysis, opioids, barbiturates, benzodiazepines, ketamine, etomidate, propfol, sedation for interventional pain management.

pain procedure (1). This review discusses the pharmacologic aspects of anxiolysis, hypnosis, and sedation in interventional pain management. A detailed review of the mechanisms of drug action and pharmacologic properties is provided.

\section{Historical Aspects}

Ethyl alcohol derived from the natural fermentation process was probably the earliest sedative-hypnotic agent. Through the ages ethanol remained a vital ingredient to various concoctions containing opium, hyoscyamus (hyoscyamine and scopolamine), and other botanicals used for therapeutic purposes.

The therapeutic usefulness of the opium poppy has been known since antiquity. Opium comprises some 20 natural alkaloids. In 1805, Serturner first isolated morphine from opium. Following Alexander Wood's invention of the hypodermic syringe and needle in 1855, intravenous use of the available anesthetic agents became possible.

In the mid-nineteenth century, bromide salts were first introduced specifically for sedation-hypnosis. The first barbiturate, barbital, was synthesized in 1903, followed by phenobarbital in 1912. Hexobarbital, the first short-acting barbiturate to be used intravenously, was introduced in 1927. The thiobarbiturates, thiopental and thiamylal, were synthesized in 1932 and thiopental was first used clinically in 1934. Meprobamate emerged in the early 1950s as a new class of minor tran- quilizers.

Chlordiazepoxide, the first of the benzodiazepine class of sedative-hypnotic tranquilizers, was introduced clinically in 1961, heralding a new era of sedative agents with more specificity for anxiolysis over other CNS depressant effects. Other benzodiazepines, including diazepam, lorazepam, and midazolam followed, which offered greater specificity and utility in providing sedation, anxiolysis, amnesia, and anesthesia.

A new era for opioid therapy was pioneered in the early 1980s with the introduction of a new class of potent, fast onset, short-acting intravenous piperidinederivative opioid agents. Fentanyl was soon followed by sufentanil, alfentanil, and most recently, remifentanil.

In 1972, ketamine, a structural analogue of the psychotomimetic agent phencyclidine, was released for clinical use as a dissociative anesthetic with multiple unique features. Etomidate was also introduced into clinical use in 1972, with the expectation it would provide minimal depression of cardiovascular and respiratory functions. Finally, in 1989, propofol (diiopropylphenol) was approved for clinical use in the United States after years of use in Europe. Its distinctive properties allow for the rapid onset and short duration of sedative and anesthetic effects. The recent trend in sedation analgesia has emphasized higher specificity of effect, greater control of action, and enhanced safety. 


\section{TERMINOLOGY}

Sedation implies calming and alleviation of anxiety, with concomitant drowsiness or sleepiness. Hypnosis, as it relates to sedation, refers to a drug-induced sleep from which the patient can be readily aroused. Analgesia entails the relief of pain, while anesthesia involves a complete loss of sensation and responsiveness to stimuli. Therefore, in common usage, the term "sedation" implies a continuum of anxiolysis, calming, sleepiness, and analgesia. However, the amount of analgesia provided during routine sedation does not supply complete relief of pain as would be expected with local or general anesthesia.

The American Society of Anesthesiologists (ASA) has defined three levels of sedation along a continuum relating to depth of effect (2). These three levels are defined as: minimal, moderate, and deep sedation (Table 1). General anesthesia is also included as an extreme level of this continuum, which is to be avoided when using sedation techniques.

During minimal sedation or anxiolysis, the patient feels relaxed and is at a wakeful level of consciousness with airway and protective reflexes intact. With moderate sedation or conscious sedation, the patient feels drowsy and has a minimally-depressed level of consciousness, yet is readily able to verbalize or respond to touch. The patient may later have no recall of events. Airway and protective reflexes remain intact. Deep sedation renders the patient unconscious, amnestic, and responsive only to verbal or noxious stimuli. There is a partial or complete loss of airway and protective reflexes.

There are varying degrees of overlap in the effects a patient may experience at each described level and it is difficult to predict which level a patient will attain with a given sedative dosage. The deep sedation level can rapidly transition into a level of general anesthesia with unconsciousness, complete loss of airway and protective reflexes, and unresponsiveness to verbal or noxious stimuli. The progression to general anesthesia deviates to the extreme of the sedation technique, placing the patient at increased risk, and necessitating the expertise and proficiency to provide airway, ventilatory, and cardiovascular support.

\section{Common Drugs}

The drugs most commonly used to impart sedation analgesia include: the barbiturates thiopental and methohexital; etomidate, propofol, and ketamine; the benzodiazepines diazepam, lorazepam, and midazolam; and the opioids morphine, meperidine, fentanyl, sufentanil, alfentanil, and remifentanil. These drugs represent a heterogeneous group of agents deriving a wide spectrum of pharmacologic effects from both selective, as well as nonspecific CNS depressant actions. Sedation analgesia techniques and regimens strive to utilize the most efficacious agents, alone or in combination, in a target-controlled manner, to provide the desired outcome (3). Drugs commonly used for sedation and analgesia, their pharmacodynamic effects and pharmacokinetic parameters are listed in Tables 2-4.

\section{Mechanisms of Action}

The various pharmacologic effects produced by the sedative analgesic drugs result from interactions at specific receptor sites within the central and peripheral nervous system. The receptor types and anatomical locations may be similar in nature or distinctly different, yet the overall effects derived from these actions serve to complement each other in the sedative analgesia scheme.

Table 1. Levels of Sedation

\begin{tabular}{|l|l|l|l|l|}
\hline Consciousness & Minimal Sedation & Moderate Sedation & Deep Sedation & General Anesthesia \\
\hline $\begin{array}{l}\text { Anake; Relaxed } \\
\text { andiolysis); coordination } \\
\text { may be impaired }\end{array}$ & $\begin{array}{l}\text { Drowsy; Sleepy; } \\
\text { Light sleep } \\
\text { (conscious sedation) }\end{array}$ & Asleep & Unconscious \\
\hline Responsiveness & $\begin{array}{l}\text { Normal response to } \\
\text { verbal and tactile stimuli; } \\
\text { understands and responds } \\
\text { to questions }\end{array}$ & $\begin{array}{l}\text { Purposeful; awakens } \\
\text { when spoken to or } \\
\text { touched; may have } \\
\text { amnesia to events }\end{array}$ & $\begin{array}{l}\text { May be unresponsive to } \\
\text { verbal commands; may have } \\
\text { purposeful movements to } \\
\text { painful stimuli }\end{array}$ & $\begin{array}{l}\text { Not arousable; Unresponsive to } \\
\text { verbal or tactile stimuli; usually no } \\
\text { movement except to painful stimuli } \\
\text { (e.g., incision) }\end{array}$ \\
\hline $\begin{array}{l}\text { Airway; Protective } \\
\text { Reflexes }\end{array}$ & Unaffected & Patent & $\begin{array}{l}\text { Airway may be impaired; } \\
\text { may require airway } \\
\text { management }\end{array}$ & $\begin{array}{l}\text { Airway often impaired (due to drug- } \\
\text { induced depression of muscle tone); } \\
\text { often requires airway support (e.g., } \\
\text { nasal airway or mask) }\end{array}$ \\
\hline Ventilation Status & Unaffected & Adequate & $\begin{array}{l}\text { Possibly inadequate; } \\
\text { supplemental oxygen } \\
\text { indicated }\end{array}$ & $\begin{array}{l}\text { Impaired; often requires support of } \\
\text { ventilation (e.g., positive pressure } \\
\text { ventilation, etc) }\end{array}$ \\
\hline $\begin{array}{l}\text { Cardiovascular } \\
\text { Function }\end{array}$ & Unaffected & Stable & $\begin{array}{l}\text { May be impaired (requires fluids and } \\
\text { vasopressor support) }\end{array}$ \\
\hline
\end{tabular}

The depth of sedation is on a continuum, from a fully awake and relaxed state to one of unconsciousness and unresponsiveness, with the need for airway support and ventilatory assistance. With deep sedation and general anesthesia, unconsciousness, which must be distinguished from sleep, may be associated with lack of protective airway reflexes, ventilatory compromise and cardiac decompensation. Sedation does not imply that the patient has adequate analgesia or anesthesia. Procedures require appropriate use of local anesthetics; use of local anesthetics can reduce the requirements for general anesthetics (e.g., propofol or inhalation agents during general anesthesia). Patients must be monitored appropriately during procedures involving sedation.

Useful references: 2 and 40. 
Table 2. Comparative spectrum of pharmacodynamic effects

\begin{tabular}{|l|c|c|c|c|c|c|c|}
\hline Drug & Anxiolysis & Sedation & Hypnosis & Analgesia & Amnesia & Anesthesia & Dependency \\
\hline Thiopental & 0 & + & + & 0 & + & + & + \\
\hline Methohexital & 0 & + & + & 0 & + & + & + \\
\hline Diazepam & + & + & + & 0 & + & + & + \\
\hline Lorazepam & + & + & + & 0 & + & + & + \\
\hline Midazolam & + & + & + & 0 & + & + & + \\
\hline Etomidate & 0 & + & + & 0 & + & + & + \\
\hline Propofol & 0 & + & + & 0 & + & + & + \\
\hline Ketamine & 0 & 0 & 0 & + & + & + D & + \\
\hline Morphine & 0 & + & + & + & 0 & + & + \\
\hline Meperidine & 0 & + & + & + & 0 & + & + \\
\hline Fentanyl & 0 & + & + & + & 0 & + & + \\
\hline Sufentanil & 0 & + & + & + & 0 & + & + \\
\hline Alfentanil & 0 & + & + & + & 0 & + & + \\
\hline Remifentanil & 0 & + & + & + & 0 & + & + \\
\hline Dexmedetomidine & 0 & + & + & + & + & S & + \\
\hline
\end{tabular}

* Possessing receptor specificity for effect; $+=$ Produces effect $; 0=$ No effect; $S=$ Anesthetic-sparing effects; $A=$ Attenuates withdrawal symptoms from barbiturates, benzodiazepines, and opioids; $D=$ Dissociative anesthetic state

\section{Barbiturates}

The action of barbiturates is mediated through the suppression of excitatory and facilitation of inhibitory synaptic neural transmissions. Barbiturates interact with the inhibitory neurotransmitter gamma-aminobutyric acid (GABA) through binding to, and activation of, the $\mathrm{GABA}_{\mathrm{A}}$ receptor subunit. $\mathrm{GABA}_{\mathrm{A}}$ receptor activation leads to increased chloride current conductance, resulting in hyperpo- larization of the postsynaptic membrane and inhibition of postsynaptic neurons. Barbiturates may also mimic the action of GABA by directly activating the chloride ion channels. Barbiturates also inhibit excitatory alpha-amino-3-hydroxy5-methyl-4-isoxazole propionic acid (AMPA) subtypes of glutamate receptors. The CNS depressant effects of barbiturates are based on the activation of inhibitory $\mathrm{GABA}_{\mathrm{A}}$ receptors and the inhibition of excitatory AMPA receptors. Within the peripheral nervous system (PNS), barbiturates selectively depress neurotransmission through the inhibition of excitatory autonomic ganglia and nicotinic cholinergic, acetylcholine receptors. Barbiturates can reversibly depress the neural activity of most excitable tissues, preferentially suppressing polysynaptic neuron responses. Barbiturates act to suppress the responses of the reticular activating sys-

Table 3. Comparative pharmacologic effects

\begin{tabular}{|c|c|c|c|c|c|c|c|}
\hline Drug & $\begin{array}{c}\text { Heart } \\
\text { Rate }\end{array}$ & $\begin{array}{l}\text { Systemic } \\
\text { Blood } \\
\text { Pressure }\end{array}$ & Ventilation & $\begin{array}{l}\text { Cerebral } \\
\text { Blood Flow }\end{array}$ & $\begin{array}{l}\text { Intra-cranial } \\
\text { Pressure }\end{array}$ & $\begin{array}{l}\text { Cerebral Metabolic Rate } \\
\text { O2 Consumption }\end{array}$ & $\begin{array}{l}\text { Histamine } \\
\text { Release }\end{array}$ \\
\hline Thiopental & $\uparrow$ & $\downarrow$ & $\downarrow$ & $\downarrow$ & $\downarrow$ & $\downarrow$ & + \\
\hline Methohexital & $\uparrow$ & $\downarrow$ & $\downarrow$ & $\downarrow$ & $\downarrow$ & $\downarrow$ & + \\
\hline Diazepam & $0 / \uparrow$ & $\downarrow$ & $\downarrow$ & $\downarrow$ & $\downarrow$ & $\downarrow$ & 0 \\
\hline Lorazepam & $0 / \uparrow$ & $\downarrow$ & $\downarrow$ & $\downarrow$ & $\downarrow$ & $\downarrow$ & 0 \\
\hline Midazolam & 0 & $0 / \downarrow$ & $0 / \downarrow$ & $0 / \downarrow$ & $0 / \downarrow$ & $0 / \downarrow$ & 0 \\
\hline Etomidate & $0 / \uparrow$ & $0 / \downarrow$ & $0 / \downarrow$ & $0 / \downarrow$ & $0 / \downarrow$ & $0 / \downarrow$ & 0 \\
\hline Propofol & $0 / \downarrow$ & $\downarrow$ & $\downarrow$ & $\downarrow$ & $\downarrow$ & $\downarrow$ & 0 \\
\hline Ketamine & $\uparrow$ & $\uparrow$ & $0 / \downarrow$ & $\uparrow$ & $\uparrow$ & $\uparrow$ & 0 \\
\hline Morphine & $\downarrow$ & $\downarrow$ & $\downarrow$ & $\downarrow$ & $\downarrow$ & $\downarrow$ & + \\
\hline Meperidine & $\uparrow$ & $\downarrow$ & $\downarrow$ & $\downarrow$ & $\downarrow$ & $\downarrow$ & + \\
\hline Fentanyl & $\downarrow$ & $\downarrow$ & $\downarrow$ & $\downarrow$ & $\downarrow$ & $\downarrow$ & 0 \\
\hline Sufentanil & $\downarrow$ & $\downarrow$ & $\downarrow$ & $\downarrow$ & $\downarrow$ & $\downarrow$ & 0 \\
\hline Alfentanil & $\downarrow$ & $\downarrow$ & $\downarrow$ & $\downarrow$ & $\downarrow$ & $\downarrow$ & 0 \\
\hline Remifentanil & $\downarrow$ & $\downarrow$ & $\downarrow$ & $\downarrow$ & $\downarrow$ & $\downarrow$ & 0 \\
\hline Dexmedetomidine & $0 / \downarrow$ & $0 / \downarrow$ & $0 / \downarrow$ & $\mathrm{U}$ & $\mathrm{U}$ & $\mathrm{U}$ & 0 \\
\hline
\end{tabular}

$\uparrow=$ Increase; $\downarrow=$ Decrease; $0=$ No change, effect; $+=$ Produces effect; $U=$ Unknown effect. 
Table 4. Comparative pharmacokinetic parameters

\begin{tabular}{|l|c|c|c|c|c|}
\hline Drug & pKa & $\begin{array}{c}\text { Partition } \\
\text { Coefficient }\end{array}$ & $\begin{array}{c}\text { Elimination } \\
\text { Half-Life } \\
\text { (hours) }\end{array}$ & $\begin{array}{c}\text { Context- } \\
\text { Volume of } \\
\text { Distribution } \\
\text { (liters/kg) }\end{array}$ & $\begin{array}{c}\text { Sensitive } \\
\text { Half-Life } \\
\text { (minutes) }\end{array}$ \\
\hline Thiopental & 7.6 & $58-63$ & 11.6 & 2.5 & 200 \\
\hline Methohexital & & & 3.9 & 2.2 & \\
\hline Diazepam & 3.4 & & $21-37$ & $1-1.5$ & \\
\hline Lorazepam & $1.3-11.5$ & & $10-20$ & $0.8-1.3$ & \\
\hline Midazolam & 6.15 & & $1-4$ & $1-1.5$ & 180 \\
\hline Etomidate & 4.2 & & $2-5$ & $2.2-4.5$ & \\
\hline Propofol & 11 & 5012 & $0.5-1.5$ & $1.8-5.3$ & 55 \\
\hline Ketamine & 7.5 & & $2-3$ & $2.5-3.5$ & \\
\hline Morphine & 7.9 & 1.4 & $1.7-3.3$ & $3-5$ & \\
\hline Meperidine & 8.5 & 32 & $3-5$ & $3-5$ & \\
\hline Fentanyl & 8.4 & 955 & $3.1-6.6$ & $3-5$ & 260 \\
\hline Sufentanil & 8.0 & 1,727 & $2.2-4.6$ & 2.5 & 30 \\
\hline Alfentanil & 6.5 & 1,145 & $1.4-1.5$ & $0.5-1.0$ & 60 \\
\hline Remifentanil & 7.3 & & $0.17-0.33$ & 0.4 & $3-4$ \\
\hline Dexmedetomidine & 7.1 & 2.89 & 2 & 1.7 & \\
\hline
\end{tabular}

tem in the brainstem that regulates the level of consciousness, as well as vital respiratory and cardiovascular functions.

\section{Benzodiazepines}

Similar to the barbiturates, the benzodiazepines enhance activity of the inhibitory neurotransmitter GABA through their binding to the GABA receptor subtype at a specific site distinct from that of the GABA binding site on the receptor (4, $5)$. This enhanced GABA-induced activity facilitates chloride ion channel conductance, leading to hyperpolarization of the postsynaptic membrane and decreased neuronal excitability. The benzodiazepine-receptor binding occurs mostly on the postsynaptic nerve membranes largely within the cerebral cortex. Unlike barbiturates, benzodiazepines do not directly activate $\mathrm{GABA}_{\mathrm{A}}$ receptors, but rather modulate GABA binding. Binding of benzodiazepines to distinctly different specific $\mathrm{GABA}_{\mathrm{A}}$ subunits is thought to be the mechanism for the various specific pharmacologic properties expressed by the benzodiazepines.

\section{Etomidate}

Etomidate is another agent also believed to enhance GABA inhibitory neural activity in a manner comparable to the barbiturates, benzodiazepines, and propofol. Unlike the barbiturates, etomidate appears to be relatively selective in its action as a modulator of the $\mathrm{GABA}_{\mathrm{A}}$ receptor.

\section{Propofol}

Propofol is thought to act through enhancing inhibitory GABA neurotransmission by decreasing the rate of GABAreceptor dissociation, thus increasing chloride ion channel conductance, hyperpolarizing the postsynaptic cell membrane, and inhibiting neuron activation. Similar to etomidate, propofol's action appears to be relatively selective for modulating the GABA A receptor. Neither etomidate nor propofol appear to modulate other ligand-gated channels at clinically applicable doses.

\section{Ketamine}

Ketamine offers pharmacologic properties and mechanisms unique to the other sedative analgesic agents. Ketamine appears to selectively depress normal functioning of the associative cortex and thalamus while enhancing activity in the limbic systemic, producing a functional disconnect between the thalamus and the limbic cortex. This action produces a cataleptic-like state of unconsciousness, termed dissociative anesthesia. Ketamine's principle action is thought to involve noncompetitive inhibition of the N-methylD-aspartate (NMDA) receptor, a glutamate-gated cationic channel selective for calcium. This NMDA glutamate receptor subtype is involved in the prolonged potentiation of synaptic responses associated with the wind-up, central sensitization phenomena. Ketamine may also interact with subgroups of endogenous opioid receptors, monoaminergic and muscarinic, cholinergic receptors. Unlike the other sedative analgesic agents, ketamine does not interact with the inhibitory GABA and glycine-gated receptors.

\section{Opioids}

Opioid mediated pharmacologic properties result from ligand binding to specific opioid receptors distributed throughout the CNS and peripheral tissues $(6,7)$. Three major types of opioid receptors have been identified: $\mathrm{mu}$ subtypes 1 and 2 and various subtypes of kappa and delta receptors; the sigma receptor is not considered to be an opioid receptor.

Opioids mimic the actions of endogenous peptide opioid receptor ligands, the endorphins, enkephalins, and dynorphins. Opioids interact at supraspinal, spinal and peripheral sites of action. Opioid-receptor activation inhibits the presynaptic release of excitatory neurotransmitters, acetylcholine and substance P. Increased potassium conductance following opioid-receptor activation results in hyperpolarization of the postsynaptic cell membrane and inhibition of neuronal response. The opioid effect is selective for nociception. Nociceptive impulses can be interrupted at various levels of the descending inhibitory (antinociceptive) modulation pathway, from the periaqueductal grey down through the nucleus raphe magnus to the dorsal horn of the spinal cord.

\section{Clinical Relevance}

The pharmacologic mechanism and site of action for the sedative analgesic agents establishes the basis for the clinically relevant aspects of these drugs in interventional pain medicine. Pharmacologic properties inherent to the physiochemical nature and structural stereoselectivity of the drug convey certain parameters as to how the drug is processed by the body and how the drug acts on the body. These respective pharmacokinetic and pharmacodynamic parameters will contribute significantly to the type and degree of effects produced by the sedative analgesic drug. Drugs within a particular class such as the barbiturates, benzodiazepines, and opioids, are all considered to have a common mechanism of action and exhibit a similar spectrum of effects. The 
particular pharmacokinetic and pharmacodynamic parameters intrinsic to an individual drug within the group will impart the distinctive quality to that drug's effects $(8,9)$.

\section{Barbiturates}

Within the barbiturate class, drugs with clinically relevant usefulness in providing sedation for various types of diagnostic or therapeutic interventional pain procedures include thiopental and methohexital. Thiopental is a parenteral thiobarbiturate, administered as a $2.5 \%$ solution with an alkaline $\mathrm{pH}$ of 10.5, making it incompatible for admixture with acidic drugs such as opioids. Methohexital, a parenteral oxybarbiturate administered as a $1 \%$ solution, is also alkaline. Both produce a dosedependent progression of CNS depression with sedation, amnesia, hypnosis, and anesthesia. They are very lipid soluble, highly protein bound, and largely non-ionized at physiologic $\mathrm{pH}$. Following a single dose intravenous (IV) administration, barbiturates rapidly accumulate in the CSF and brain tissues leading to a fast onset of effects, usually within 30 seconds. The dose and rate of administration are two primary determinants of plasma concentration affecting drug diffusion into the CSF. The roughly 20 -minute duration of action is largely determined by a rapid redistribution of drug from the vessel rich groups of tissues (brain) to peripheral compartments in muscle and fat, which lowers plasma and brain drug concentrations. A more prolonged hepatic metabolism following first-order kinetics and renal excretion, accounts for the elimination of drug. Following repeat bolus dosing or continuous infusion administration with saturation of all tissue sites, the termination of drug effects becomes more dependent on hepatic metabolism than redistribution.

Methohexital is twice as potent as thiopental. Its onset of action is equivalent to thiopental, yet its duration of action is half as long. Methohexital has fewer cumulative effects and a faster recovery than thiopental following repeat boluses or continuous infusion. Cardiovascular effects of barbiturates are greatly influenced by the patient's intravascular volume status, basal autonomic activity, and cardiac functioning.

Barbiturates depress the central medullary vasomotor center resulting in a decreased cardiac output, systemic arterial pressure, and peripheral vascular resistance. A reflex tachycardia in response to hypotension normally maintains cardiac output along with an increased myocardial contractility through compensatory baroreceptor reflexes. At an equivalent dose, methohexital produces less hypotension by allowing a greater heart rate response to decreased blood pressure by reducing baroreceptor reflex sensitivity. Hypotension produced by the barbiturates is reflected in reduced hepatic and renal blood flows. Barbiturates cause a dose-dependent depression of the central medullary respiratory center resulting in a decrease in respiratory rate, tidal volume, and apnea with a decreased ventilatory responsiveness to hypercapnia and hypoxia. Airway reflexes are not sufficiently depressed, thus noxious airway stimuli can induce laryngospasm and bronchospasm. Laryngospasm and hiccoughing occur more often with methohexital.

Barbiturates constrict cerebral vasculature resulting in decreased cerebral blood flow (CBF), cerebral blood volume, and intracranial pressure (ICP). The decrease in systemic arterial pressure is usually less than the reduction in ICP, thus cerebral perfusion and compliance are increased. A proportional decrease in cerebral metabolic oxygen consumption $\left(\mathrm{CMRO}_{2}\right)$ and $\mathrm{CBF}$ allows a lower ICP. $\mathrm{CMRO}_{2}$ can be maximally decreased (55\% of normal) by barbiturates when the electroencephalogram (EEG) becomes isoelectric (burst-suppressive pattern) (10). In contrast to decreasing EEG electrical activity, methohexital tends to activate epileptic foci. Barbiturates in general possess anticonvulsant activity. Subhypnotic doses of barbiturates can cause a paradoxical state of excitement and disorientation.

Barbiturates are not analgesic and may even lower the threshold for pain. Barbiturates do not provide muscle relaxation and can cause involuntary muscle contractions or tremors. Methohexital is associated with causing myocloniclike muscle contractions and diaphragmatic hiccoughing. Tolerance to the barbiturate sedative-hypnotic effects tends to develop with repeated and prolonged use. There is considerable potential for developing physical dependence, as well as a withdrawal syndrome upon discontinuation of drug use.

\section{Benzodiazepines}

The benzodiazepines of clinical relevance for use within a sedation analgesia format include diazepam, midazolam, and lorazepam. All of these agents can be administered parenterally. Oral dosage forms are available for diazepam and lorazepam, while the parenteral form of midazolam can be used orally or intranasally for effect. Diazepam and lorazepam are insoluble in water and their formulations with propylene glycol cause painful irritation on injection. Midazolam is water soluble in an acidic solution $(\mathrm{pH} \mathrm{3})$, with minimal irritation. All agents are lipid soluble and highly protein bound. As a sedative, midazolam is twice as potent as diazepam and half as potent as lorazepam, while lorazepam itself is six times as potent as diazepam. At physiologic $\mathrm{pH}$ (7.4), midazolam undergoes an intramolecular reconfiguration, which increases its lipid solubility (11). Following a single IV sedative dose, the onset of effect for midazolam is rapid, 30 to 60 seconds, gaining fast entry into the CSF. Its duration of effect, which ranges from 15 to 80 minutes, is terminated by redistribution of midazolam to peripheral tissue sites and rapid metabolic clearance. Diazepam's lipid solubility is similar to that of midazolam and exhibits a rapid onset of effect within 60 seconds and a longer duration of effect, lasting one to six hours. Diazepam undergoes hepatic conversion to an active metabolite with a slow metabolic clearance. The lipophilicity of lorazepam is less than either midazolam or diazepam such that its onset of effect is slower, within one to two minutes, while its duration of effect is much longer, extending six to 10 hours, due to a slower metabolic clearance (12). While the volumes of distribution are similar amongst the benzodiazepines, the clearance rates for diazepam and lorazepam are much slower than for midazolam. The slower metabolic clearance rates are reflected in longer context-sensitive halftimes for diazepam and lorazepam, which impede their use in short-term continuous infusion regimens.

The spectrum of effects imparted by the benzodiazepines include: anxiolysis, sedation, amnesia, hypnosis, anticonvulsant, myorelaxation, and anesthesia. The potency and efficacy exhibited by the various benzodiazepines with regard to these characteristic effects will depend upon their individual inherent affinity for the GABA receptor subtypes (4), as well as 
their degree of receptor binding.

Benzodiazepines produce a dose-dependent cardiovascular depression usually expressed as a reduced peripheral vascular resistance with decreased arterial blood pressure. Depressant effects are more pronounced in the presence of preexisting cardiac disease, hemodynamic instability, or in conjunction with opioid, propofol, or barbiturate administration. Midazolam tends to reduce peripheral vascular resistance to a greater extent than diazepam and may exert a vagolytic effect on heart rate. The respiratory depressant effects of the benzodiazepines are dose-dependent and result from a depressed central ventilatory response to hypercapnia and hypoxia. Depressant effects tend to be greater for midazolam than diazepam or lorazepam in equipotent doses and are accentuated in the presence of respiratory disease or with the concomitant administration of other sedative-hypnotics or opioids. Unlike the barbiturates, benzodiazepines can suppress upper airway reflexes. Benzodiazepines decrease $\mathrm{CMRO}_{2}$ and $\mathrm{CBF}$ while maintaining a normal $\mathrm{CBF} / \mathrm{CMRO}_{2}$ ratio similar to the barbiturates and propofol, although not to the same extent. A burst-suppressive, isoelectric EEG can not be produced by benzodiazepines, indicating a ceiling effect to decreases in $\mathrm{CMRO}_{2}$ induced by benzodiazepines.

Little or no change in ICP is noted with benzodiazepines. The muscle relaxant effects produced by benzodiazepines are mediated centrally through the spinal cord and not peripherally at the neuromuscular junction. The potency of the amnestic effects for the benzodiazepines is greater than their respective sedative effects, resulting in a longer duration of amnesia than sedation. Lorazepam has four times the amnestic potency of midazolam. Like other sedative-hypnotic drugs, the benzodiazepine exhibit tolerance to their effects with repeated, long-term use and have the potential for developing physical dependence, as well as a withdrawal syndrome, upon abrupt discontinuation of use.

In contrast to other sedative-hypnotic drugs, there is a specific antagonist agent for the benzodiazepine class of drugs. Flumazenil, a structural analog of the benzodiazepines, has a high affinity for benzodiazepine-receptor ligand binding with minimal intrinsic activity Flumazenil acts as a competitive antago- nist to the benzodiazepine agonist at the receptor site. The degree to which agonist activity is reversed or blocked by flumazenil is dependent on the concentration of flumazenil present relative to that of the agonist. Flumazenil is short-acting with an elimination half-life of one hour. Roughly 45 to 90 minutes of antagonism results from a single intravenous dose. For prolonged antagonist effects, repeat bolus dosing of flumazenil or a continuous infusion can be utilized. While it can be expected and anticipated that all benzodiazepine-induced effects will be blocked by flumazenil, respiratory depression is not completely reversed and the effects on $\mathrm{CBF}$ and $\mathrm{CMRO}_{2}$ do not change. When flumazenil is administered in the presence of chronic, long-term benzodiazepine usage, an acute withdrawal syndrome can be precipitated that is stereotypic of the sedative-hypnotics.

\section{Propofol}

Propofol is a chemically distinct sedative-hypnotic agent that is insoluble in water and is formulated as an egg lecithin emulsion for IV use. Pain on injection is common and can be ameliorated by prior administration of $1 \%$ lidocaine or an analgesic. Propofol is highly lipid soluble, and after a single intravenous dose, produces a rapid onset of effect within 30 to 45 seconds, and rapid effect-site equilibration time similar to that of thiopental. Duration of effect is short, 5 to 10 minutes, largely due to redistribution to peripheral tissues. Due to the extensive variability in the therapeutic window for dosing propofol, it is best titrated to effect (13).

Propofol undergoes extensive hepatic metabolism to inactive metabolites that are excreted renally. The metabolic clearance rate for propofol is also rapid, 10 times that of thiopental, which exceeds the rate of hepatic blood flow, indicating possible extrahepatic routes of metabolism such as the lung. Context-sensitive half-time for propofol is short, less than 40 minutes, and does not appear to be significantly affected by the duration of a continuous infusion. Prompt recovery from effect results after even prolonged infusions. While the therapeutic index of 3.4 for propofol is comparable to that of thiopental at 3.9, recovery is faster after propofol than after thiopental (14). This differential is further extended with the administration of repeat bolus doses or continuous infusions.

Propofol produces a dose-dependent depression of the cardiovascular system consisting of both direct myocardial depression and decreased peripheral vascular resistance. This depressant effect is greater than that of thiopental, given comparable dosages. Propofol inhibits sympathetically mediated vasoconstriction of peripheral vasculature and alters the baroreflex mechanism, resulting in less increase in heart rate for a given fall in systemic blood pressure. This cardiovascular depression and hypotension are exaggerated in the presence of hypovolemia and preexisting cardiac disease.

Propofol produces profound respiratory depression, decreasing minute volume and producing episodes of apnea to a greater extent than does thiopental. Propofol infusions at subanesthetic doses will inhibit ventilatory responses to hypercapnia and hypoxia. Propofol depresses laryngopharyngeal reflexes to a greater degree than barbiturates, thus facilitating upper airway manipulations. Propofol can decrease CBF, ICP, and $\mathrm{CMRO}_{2}$. Because of propofol's cardiovascular depressant effects, mean arterial pressure must be supported when treating elevated ICP in order to prevent critical reductions in CPP.

Propofol does not alter cerebrovascular autoregulation. Propofol possesses anticonvulsant activity; however, neuroexcitatory events such as myoclonus, opisthotonus, and hiccoughing have been associated with propofol administration. Subhypnotic doses of propofol can provide antiemetic and antipruritic effects. Propofol is not analgesic. Tolerance does not develop to propofol following repeated dosing or long-term infusion.

\section{Etomidate}

Etomidate is also chemically unrelated to other sedative-hypnotic or anesthetic agents. Its carboxylated imidazole structure is unstable in water and is thus solubilized for IV use in propylene glycol. This formulation, with a $\mathrm{pH}$ of 6.9 , is associated with pain on injection and thrombophlebitis. Irritation may be minimized by prior injection of lidocaine or analgesic. Etomidate is very lipid soluble and highly protein bound. At physiologic $\mathrm{pH}, 99 \%$ of the drug is non-ionized. Thus, it has a rapid onset of effect following a single intravenous dose with redistribution accounting largely for its rapid emergence time. 
Etomidate undergoes hepatic and plasma esterase hydrolysis to an inactive metabolite. Metabolic clearance for etomidate is five times greater than for thiopental.

In contrast to the barbiturates and propofol, etomidate exerts minimal effects on the cardiovascular system. A slight reduction in peripheral vascular resistance might be noted, while the cardiac output usually remains unchanged. Etomidate has minimal respiratory depression and rarely produces apnea. Like the barbiturates and propofol, etomidate decreases $\mathrm{CMRO}_{2}, \mathrm{CBF}$, and ICP. Since it has minimal cardiovascular effects, elevated ICP can be treated while maintaining CPP.

Etomidate produces EEG changes similar to the barbiturates, however it can also produce epileptic-like EEG potential in the presence of an underlying seizure disorder. Excitation phenomena and myoclonus have been associated with etomidate, as well as a high incidence of postoperative nausea and vomiting.

Etomidate is not analgesic. Etomidate causes a dose-dependent, reversible suppression of adrenocortical steroidogenesis through enzyme inhibition. Suppression is noted after a single dose, but is probably not clinically significant unless etomidate is infused for prolonged periods.

\section{Ketamine}

Ketamine, a structural analog of phencyclidine, is unique in comparison to other sedative-hypnotic and anesthetic agents in its ability to provide both amnesia and analgesia at subanesthetic doses (15-17). Ketamine is available as a racemic mixture of two stereoisomers. In contrast to propofol and etomidate, ketamine is water soluble in formulation and is not associated with pain on injection. Ketamine is highly lipid soluble, yet less protein bound than other sedative analgesic agents. Following IV administration, ketamine attains a rapid onset of effect with redistribution to peripheral compartments and hepatic metabolism, accounting for recovery. Non-parenteral (enteral, sublingual and nasal) routes of ketamine administration require larger doses and show delayed onset, reduced bioavailability, limited absorption, and extensive firstpass metabolic effects.

Ketamine is biotransformed by hepatic cytochrome P450 enzymes (CYP) into several metabolites, some retaining activity with end products being excreted renally. Ketamine exhibits a high hepatic extraction ratio and a short elimination clearance rate. Unlike other sedative-hypnotic and anesthetic agents, ketamine is devoid of cardiorespiratory depressant effects.

Ketamine's dose-dependent increase in heart rate, arterial blood pressure, and cardiac output are medicated through sympathetic nervous system stimulation and increased catecholamine release. This increased cardiac workload can affect myocardial oxygen supply and demand and can also be detrimental in the presence of ischemic heart disease and hypertension. Tachycardia and hypertension can be managed through the concomitant use of a benzodiazepine, barbiturate, or opioid agent. This indirect sympathomimetic effect tends to predominate over ketamine's potential for direct myocardial depressant effects. Loss of sympathetic tone such as in shock-like states and catecholamine deletion can, however, result in hypotension with ketamine administration.

Ketamine does not significantly depress respiratory functions. In fact, ketamine can improve pulmonary compliance and provide bronchodilation, making it a favorable agent to use in the presence of reactive airway disease. Administration of induction doses, especially in conjunction with opioids, can result in apnea. Protective upper airway reflexes are usually maintained. Ketamine does increase salivary and bronchial gland secretions, mediated through central cholinergic stimulation. This effect can be attenuated with the use of an anti-sialogogue agent. Ketamine causes cerebral vasodilation, increasing CBF and ICP. Thus, ketamine should be avoided in conditions of decreased intracranial compliance. Emergence phenomena consisting of psychotomimetic effects, hallucinations, delirium, and vivid dreams occur following recovery from ketamine. The incidence of these reactions can be up to $30 \%$, occurring more commonly in adults. Pretreatment with benzodiazepines can minimize these effects.

\section{Opioids}

Drugs within the opioid class that exhibit clinical significance for use within a sedative analgesic scheme include morphine, meperidine, fentanyl, sufentanil, alfentanil, and remifentanil. These opioids produce their effects mostly though interactions with the mu receptor subtypes. The different pharmacokinetic properties of these agents will largely determine the onset, duration, and disposition of the drug effect, whereas the drug's receptor specificity and affinity will determine the type and quality of effect. In terms of their pharmacokinetic profiles, certain opioids within this group lend themselves well to use in short-term continuous infusions or repeated bolus dosing when the goal is to provide a rapid onset and offset of effects. Pharmacokinetic profiles exhibiting high lipid solubility and rapid clearance rates contribute towards low context-sensitive half-times. Context-sensitive half-time indicates the time necessary for drug plasma concentration to decrease by $50 \%$ following discontinuation of an infusion of a particular duration $(18,19)$.

Morphine, considered the prototypic mu agonist, is poorly lipid soluble and highly ionized at physiologic $\mathrm{pH}$. Following a single IV dosing, onset of effect is slow, lagging behind peak plasma concentrations. CSF concentrations of morphine peak at 15 to 30 minutes after administration and decline slower than plasma levels, prolonging the duration of effect. Thus, plasma concentrations of morphine do not closely correlate with its pharmacologic activity. Less than $0.1 \%$ of IV morphine enters the CNS; most accumulates in the liver, kidneys, and muscle. Morphine undergoes hepatic and extrahepatic, renal, glucuronidation metabolism. Unlike fentanyl, morphine does not undergo significant first-pass uptake in the lungs. One metabolite, morphine6-glucuronide, is active and has a greater potency and duration than the parent morphine.

Meperidine is a synthetic phenylpiperidine opioid agonist at mu and kappa receptors. Meperidine has about onetenth the potency of morphine. Following IV administration it has a faster onset and shorter duration of effect than morphine. Clearance of meperidine depends on extensive hepatic metabolism and urinary elimination. Normeperidine is an active metabolite with a long elimination halflife, possessing analgesic and CNS stimulant effects. In the presence of decreased renal function or with prolonged meperidine use, normeperidine can accumulate, causing myoclonus and seizures.

Fentanyl is also a synthetic phenylpiperidine opioid that is 75 to 100 times 
more potent than morphine. Following a single dose IV administration, fentanyl exhibits a rapid onset and short duration of effect. Both the higher potency and rapid onset reflect fentanyl's greater lipid solubility compared to that of morphine. Fentanyl's short duration of effect following a single dose results from its rapid redistribution to inactive peripheral tissues. Roughly $75 \%$ of the initial fentanyl dose undergoes first-pass pulmonary uptake. With multiple repeat dosing or continuous infusion, inactive tissue sites become saturated and plasma clearance and duration of effects can be prolonged. Fentanyl from peripheral tissue compartments returns to the plasma as fentanyl and is eliminated by hepatic metabolism, which slows the rate of decrease in plasma concentration and increases the context-sensitive half-time of fentanyl. Fentanyl undergoes extensive hepatic N-demethylation metabolism and renal excretion. A primary metabolite, norfentanyl, retains a lesser analgesic potency. Despite fentanyl's short duration of effect, its elimination half-time is longer than morphine. This is can be attributed to fentanyl's larger volume of distribution (Vd), secondary to its greater lipid solubility and tissue uptake compared to morphine's low lipophilicity. Plasma concentrations of fentanyl are maintained by slow reuptake from inactive tissue reservoir sites.

Sufentanil is a thienyl analogue of fentanyl, with an analgesic potency five to 10 times that of fentanyl, which parallels a greater affinity of sufentanil for the opioid receptor. A single IV dose results in a rapid onset of effect, similar to fentanyl, with equivalent CNS effect-site equilibration times. Single dose effects are of short duration due to prompt redistribution to inactive tissue sites. Sufentanil undergoes substantial first-pass pulmonary uptake to an extent similar to fentanyl. Sufentanil is rapidly metabolized by $\mathrm{N}$-dealkylation and O-demethylation, forming some weakly active metabolites. Both hepatic clearance and renal excretion are important for sufentanil clearance. Cumulative drug effects can accompany repeat boluses or extended infusions. The context-sensitive half-time for sufentanil is less than that for fentanyl or alfentanil, primarily due to sufentanil's larger Vd (14). This provides sufentanil with a more favorable recovery profile.

Alfentanil is a tetrazole analogue of fentanyl possessing only one-fourth to one-tenth the potency of fentanyl (12). Alfentanil has lower lipid solubility than either fentanyl or sufentanil. After single dose intravenous administration, alfentanil demonstrates a more rapid onset of effect than fentanyl or sufentanil, with a CNS effect-site equilibration time of 1.1 minutes, compared with fentanyl and sufentanil at 6.8 and 6.2 minutes, respectively (14). Alfentanil is roughly $90 \%$ non-ionized at physiologic $\mathrm{pH}$ due to its low $\mathrm{pKa}$, thus permitting ready access to the CNS and rapid plasma-brain equilibration. The elimination half-time for alfentanil is shorter than that of fentanyl or sufentanil. Alfentanil's smaller Vd, relative to fentanyl and sufentanil, contributes to a longer context-sensitive half-life for alfentanil, since distribution of alfentanil from the plasma to tissue reservoirs is insignificant. Alfentanil's short duration of effects, 15 to 20 minutes, reflects its rapid redistribution and elimination parameters. Alfentanil undergoes rapid hepatic clearance and metabolism with renal excretion.

Remifentanil is also a congener of fentanyl, possessing selective mu opioid receptor agonist activity. Remifentanil has an analgesic potency similar to fentanyl and roughly 20 times that of alfentanil, yet shares a similar effect-site equilibration time as alfentanil, about six times as fast as fentanyl and sufentanil. Structurally, remifentanil is unique to all the other opioids in containing a methyl ester side chain linkage, which makes it vulnerable to nonspecific tissue and plasma esterase hydrolysis to inactive metabolites. Thus, a single dose IV administration of remifentanil results in both a rapid onset and offset of effects. Remifentanil's small Vd and rapid clearance account for its short-lived effects with an elimination half-time of roughly six minutes. The context-sensitive half-time of about four minutes for remifentanil is much shorter than that for fentanyl, sufentanil, and alfentanil, and unlike the others it is not dependent on the duration of infusion. Remifentanil's pharmacokinetic properties provide the advantages of rapid controllable titratability to effect, short duration, lack of cumulative effects even in the presence of renal or hepatic dysfunction, and a rapid, controllable, predictable recovery.

\section{Pharmacodynamic Effects of Opioids}

The desirable and therapeutic spectrum of effects provided in a dose-dependent fashion by the opioids includes an- algesia, sedation, euphoria, hypnosis (narcosis), altered consciousness, and anesthesia. All of the opioids produce a similar spectrum of systemic effects that may be viewed as ancillary or side effects in deference to the desired, primary effects. Cardiovascular effects of opioids are doserelated and can involve bradycardia and vasodilation, from a reduction in resting sympathetic tone (an effect on the hypothalamus), causing hypotension. Morphine may cause vasodilation indirectly by causing the release of histamine. Opioids can depress medullary vasomotor centers, decreasing central sympathetic tone and reducing preload and afterload. Carotid sinus baroreceptor reflex control of heart rate can be depressed by fentan$\mathrm{yl}$ and its analogue opioids. Hypotension also can result from central stimulation of vagal nuclei in the medulla, or a direct depressant effect on the sinoatrial node. Meperidine has anticholinergic properties and usually no related bradycardia. Fentanyl, sufentanil, alfentanil, and remifentanil all produce bradycardia to a much greater extent than morphine.

Morphine and meperidine are associated with histamine release, which is influenced by dose and rate of administration. Opioid-induced histamine can cause bronchospasm in reactive airway-sensitive patients. Even in large doses, fentanyl and its congeners do not cause histamine release.

Opioids produce a dose-related respiratory depression by direct effect on central brainstem ventilatory centers mediated through mu 2 receptors. Respiratory depression is characterized by a decreased responsiveness to hypercapnia, elevating the apneic threshold, and diminishing hypoxic drive. Ventilatory mechanics are manifested by decreased frequency of breathing, compensated by increased tidal volume. At sufficiently high doses, tidal volume decreases and apnea can occur. The concomitant use of barbiturates and benzodiazepines can have a synergistic effect on the respiratory depressant effects of opioids. Under conditions of normocarbia, opioids generally reduce $\mathrm{CBF}$, ICP, and $\mathrm{CMRO}_{2}$, however, they do so to a much lesser extent than the barbiturates and benzodiazepines. Opioids usually show no EEG evidence of seizure activity, except potentially at high doses.

Neurotoxicity from the accumulation of the meperidine metabolite, normeperidine, can cause myoclonus and 
seizures. Opioid-induced muscle rigidity represents a centrally mediated, generalized hypertonicity of body skeletal muscle, which can impair ventilation. It is most often associated with fentanyl and its congeners following the rapid administration of large doses. Opioid-induced nausea and vomiting results from a direct stimulant effect on the medullary chemoreceptor trigger zone of the area postrema. No apparent dose-dependent relationship seems to exist for this effect. Opioids act on peripheral receptors in gut and genitourinary tract smooth muscle to stimulate tonic contractions and decrease propulsive peristaltic contractions. Resultant side effects include delayed gastric emptying, biliary colic, constipation, ileus, and urinary retention.

Like the barbiturates and benzodiazepine, the development of tolerance and physical dependence results from repeated dosing and prolonged administration of opioids agonists. Tolerance is usually manifested early as a decrease in the duration of effect followed by a decrease in intensity of effect. Cross-tolerance develops between all opioids, although it is often incomplete. Acquired tolerance may take two to three weeks to develop to analgesic doses of morphine. Opioid-induced tolerance develops more rapidly to depressant effects such as analgesia and respiratory depression, and slowly towards stimulant effects like constipation and miosis. Following the development of tolerance, a state of physical dependence is induced whereby a stereotypical opioid withdrawal syndrome will result upon discontinuation of an opioid drug. Onset of withdrawal and abstinence symptoms typically occurs within 15 to 20 hours and can last 10 to 14 days. Following withdrawal, tolerance to the opioid effects is lost.

Like the benzodiazepine class of drugs, there exists specific drug antagonists to the opioid mu agonist effects. These antagonist agents have a high affinity for the opioid receptor, yet lack the intrinsic activity to activate it. Naloxone and naltrexone, two such opioid antagonists, are structural analogs of morphine. They display competitive inhibition and displacement of opioid agonists at the receptor level. Administered intravenously, naloxone promptly reverses opioid-induced effects and respiratory depression, as well as analgesia. Naloxone has a short duration of action, 30 to 45 minutes, and a rapid elimination half-time, 60 to $90 \mathrm{~min}$ - utes. It may be necessary to repeat naloxone dosing or administer a continuous infusion for prolonged opioid antagonism. Side effects accompanying the reversal of opioid effects may include nausea, vomiting, tachycardia, hypertension, cardiac dysrhythmias, and pulmonary edema. Acute opioid withdrawal symptoms can also be precipitated in the presence of opioid physical dependence. Adverse effects can be minimized by careful titration of naloxone in terms of dose and rate of administration. In contrast to naloxone, naltrexone is effective orally and produces sustained antagonism for as long as 24 hours.

\section{Side Effects and Complications}

Effects other than the primary desired effect of the sedative analgesic agents may be considered adverse or side effects. These usually represent an extension of the drug's pharmacologic spectrum of effects. Many of these effects have previously been alluded to under pharmacologic aspects. Complications from a drug are usually unexpected and result from a hypersensitivity type of reaction or a drug interaction with a drug from within the same class or from an entirely different class. Drug interactions can be antagonistic or synergistic in nature. Additionally, the use of a drug under a certain set of conditions, subserving a potential for harm or complication, warrants a contraindication to its use.

Excessive ventilatory or cardiovascular depression as an extension of the pharmacologic effects of the sedative analgesics can be the result of a dose-dependent response or from the combined effect of a drug interaction. An additive drug interaction occurs when the combined effects of drugs working through a common mechanism of action produces a sum total of effect. A synergistic interaction involves the combined effect of drugs mediated through different mechanisms yielding a response greater than simple sum total. Antagonistic interactions result in an effect less than that of one drug alone, or essentially a reversal of a drug's effect. The concurrent use of a barbiturate and benzodiazepine would be expected to produce additive depressant effects. However, co-administration of a sedative-hypnotic with an opioid would result in marked synergist depressant effects. Ventilatory depressant effects of the opioids can also be accentuated by the use of tricyclic anti- depressants, phenothiazines, monoamine oxidase inhibitors (which are contraindicated with meperidine), and amphetamines. The use of beta and alpha adrenergic blocking agents, such as metoprolol and terazosin, can unmask the direct myocardial depressant effects of ketamine. Drug interactions can produce effects through altering biotransformation processes that either induce or inhibit drug metabolism or clearance. Plasma protein binding displacement can increase the fraction of a drug available for activity. The barbiturates can induce certain hepatic enzymes and inhibit others. Cimetidine is known to reduce diazepam metabolism, and erythromycin can inhibit midazolam metabolism through actions on hepatic microsomal enzymes. Plasma levels of fentanyl and alfentanil can be increased with the concurrent use of propofol, while the concomitant use of fentanyl can elevate plasma concentrations and extend the elimination of etomidate.

Allergic drug reactions can be either anaphylactic or anaphylactoid in nature. The anaphylactic type involves an antibody-antigen interaction mediated by a type I hypersensitivity reaction that is not dose-related. The anaphylactoid type does not involve an antibody-antigen contact, but rather a drug-induced histamine release from mast cells or drug-activated complement response. Those hypersensitivity reactions involving sedative analgesic drugs are mostly of the anaphylactoid type. Thiopental, unlike methohexital, shows a greater potential to evoke mast cell release of histamine. The incidence of hypersensitivity reaction with thiopental is $1 / 14,000$ versus $1 / 7,000$ for methohexital. Gross estimates for other sedative analgesic agents include etomidate at roughly $1 / 450,000$ and propofol 1/100,000 (14). Etomidate, propofol, and ketamine do not induce the release of histamine. Propofol's allergenic properties are attributed to its structural phenyl nucleus and diisopropyl side chain. Both morphine and meperidine evoke histamine release, but none of the fentanyl analogues cause histamine release, even at high doses. In terms of the histamine release potential of certain sedative analgesics, the risks for adverse reactions would be increased in the presence of reactive airways, asthma, and chronic atopy.

There are few absolute contraindications to the use of certain of the sedative analgesics. Known hypersensitivity 
reaction to a drug or its congeners seems obvious. Relative contraindications rely on clinical judgment and a risk-to-benefit analysis. The barbiturates and etomidate should not be used in the presence of the various porphyria-predisposed conditions. Due to it ability to activate epileptic foci, etomidate should be used with precaution, if at all, in the presence of epileptiform disorders. Ketamine should be avoided in the presence of systemic and pulmonary hypertension, ischemic, and valvular heart disease, and conditions of decreased intracranial compliant or increased intraocular pressure. Consideration should be given to the use of ketamine in the presence of psychiatric illnesses as a result of its propensity to produce psychotomimetic effects during recovery. The incidence of emergence delirium with ketamine in adults is 10 to $30 \%$, and is substantially lower in pediatrics patients; it may be dose-dependent. Benzodiazepines, diazepam, and midazolam should be avoided in the presence of acute narrow angle glaucoma and untreated open angle glaucoma.

Sedative analgesic use in pregnancy is controversial. Opioids appear to be relatively safe and are given a Class $B$ rating (no evidence of risk in humans) on the FDA Classification of Teratogenicity. The barbiturates thiopental and methohexital are also Class B. The benzodiazepines and etomidate are rated $\mathrm{C}$ (risk cannot be ruled out). When used in the first trimester, diazepam has been shown to be associated with cleft lip, cleft palate, and congenital hernias.

Precautions need to be taken regarding the use of strict aseptic techniques in handling propofol. Most propofol formulations do not contain bacteriostatic additives and propofol significantly supports bacterial growth. Every effort should be taken to prevent contamination of propofol vials and syringes. Unused propofol should be discarded six hours after open exposure. Finally, unlike the volatile anesthetics, the barbiturates, benzodiazepines, etomidate, propofol, ketamine, and the opioids do not trigger malignant hyperthermia.

\section{Relevant Aspects in Interventional Pain Management}

The sedation analgesia drugs afford the means by which diagnostic and therapeutic interventional pain procedures can be performed in a manner conducive to compassionate patient care. The continuum of sedation analgesia allows various levels of relief from anxiety, stress, discomfort, and recall associated with an invasive interventional process. The pharmacologic properties and effects of these drugs are most clinically beneficial when they are applied in a manner that best utilizes their advantages to the needs of the situation.

\section{Goals}

Sedation analgesia objectives are to serve the needs of the patient and the requirements of the procedure (20). Procedural variables to consider are the nature of the procedure, its degree of invasiveness, and previous experience with a patient's tolerance or discomfort. Also important considerations are the overall duration of the procedure and the extent to which the need for patient immobility, cooperation, or participation is expected. Patient considerations include the following: age, especially whether pediatric or elderly; health status, particularly in terms of cardiopulmonary functioning and hepatic and renal functions; ability to cooperate and communicate; pregnancy status; level of maturity and capacity to understand all that the procedure entails; and known drug allergies or past reactions. These patient variables will require a focused examination and assessment by the practitioner. Once the procedural aspects have been determined and the patient is informed and evaluated, then the sedation analgesia regimen can be planned.

The goal of the sedation analgesic treatment plan is to maintain a therapeutic level of medication in order to attain the desired plane of sedation along the continuum throughout the procedure period. This treatment is best provided using the IV route for drug administration, carried out using single or repeat bolus dosing, bolus injection followed by a continuous infusion, or possibly by a target controlled, computer-assisted continuous infusion (21-23). The computer-assisted infusion incorporates a drug's pharmacokinetic data profile with an infusion device, which takes the set therapeutic plasma level, injects a bolus dose, and follows with a set continuous infusion. This computerized mode can potentially provide the most constant plasma concentrations.

\section{Variables}

Selection variables for the sedation analgesic agent(s) involve consider- ation of the drug's inherent pharmacologic properties, and the preferable pharmacokinetic and pharmacodynamic characteristics, as previously discussed. Rate of onset and recovery, mode of elimination, and balance between those desirable and adverse effects are all primary factors (24). Within this context, consideration is made for the inherent limitations in pharmacologic spectrum of effect for the various sedative analgesic drugs. The sedativehypnotic agents, such as the barbiturates, benzodiazepines, etomidate, and propofol, are capable of producing a dose-dependent spectrum consisting of anxiolysis, sedation, amnesia, hypnosis, and anesthesia. Ketamine is unique. It creates a cataleptic-like state of calming sedation, analgesia, amnesia, hypnosis, and anesthesia. Similar to the volatile anesthetics, ketamine is a complete intravenous anesthetic. The opioids, to varying degrees of effect, produce a spectrum consisting of sedation, analgesia, hypnosis (narcosis), and anesthesia.

\section{Pharmacologic Agents}

A variety of sedative analgesic agents are available to assist in providing interventional pain procedures (25). Both thiopental and methohexital provide a rapid onset of effect and prompt awakening after a single IV dose, reflecting rapid redistribution from CNS to peripheral inactive tissues. However, following multiple repeated doses or protracted infusion, the context-sensitive half-time is prolonged, which does not lend itself well to short procedures requiring a faster recovery.

Within the benzodiazepine group, midazolam exhibits both a faster onset and offset of effect after a single IV dose than either diazepam or lorazepam; it also provides a greater degree of amnesia. Following multiple IV boluses or infusion, midazolam has a shorter context-sensitive half-time than diazepam or lorazepam due to its rapid hepatic clearance.

Following a single IV dose, etomidate also displays a rapid onset and offset of effects and quick awakening owing to its fast redistribution and rapid esterase hydrolysis. Etomidate's context-sensitive half-life is less than thiopental and is not affected by the duration of infusion. Due to its lack of cardiovascular depressant effect, etomidate would be a good alternative in the presence of cardiovascular dysfunction. Drawbacks might include pain- 
ful injection and myoclonus.

Propofol's fast onset and offset of effect following a single IV bolus, coupled with its short context-sensitive halflife with even prolonged infusions make it readily titratable to effect $(23,26,27)$. Similar to the barbiturates, propofol's pediatric dosage requirements are somewhat higher, while elderly patients will require fewer drugs $(28,29)$. In addition to the advantages of fast onset, prompt recovery with minimal residual sedation, propofol possesses antiemetic and antipruritic properties. Painful injection and lack of analgesia are shortcomings.

Ketamine exhibits a rapid onset of effect following a single IV dose, however full recovery is delayed and is even more prolonged after repeat boluses or infusion. Ketamine provides intense analgesia and amnesia at subanesthetic doses (30). Ketamine's sympathetic stimulant effect needs to be considered in the presence of cardiovascular dysfunction. The emergence delirium and excitation associated with ketamine can be attenuated with the co-administration of a benzodiazepine. As an aside, it is common for anesthesia professionals to combine ketamine with propofol (a mix called "Ketofol") in order to derive therapeutic benefits from the advantages of each drug, as well as to minimize their disadvantages.

The opioid class adds another dimension to the sedative analgesic regimen (30). Both morphine and meperidine administered IV exhibit slower onset and longer duration of effects than the fentanyl analogues, making them less titratable. Their higher association with nausea and vomiting, as well as histamine release, also make them less desirable. A single IV dose of fentanyl has a faster onset and shorter duration than morphine. However, with multiple repeated doses or long continuous infusions, fentanyl's inactive redistribution sites become saturated. Coupled with its slow clearance rate, fentanyl's contextsensitive half-time becomes prolonged. Sufentanil exhibits a rapid onset and offset of effects after a single IV dose with a context-sensitive half-time shorter than alfentanil. Alfentanil shows a rapid onset and offset of effects following IV administration. The context-sensitivity half-times for both sufentanil and alfentanil are short and infusion duration time dependent. Remifentanil also displays a rapid onset and offset of effects following IV dosing (31); it possesses an extremely short context-sensitive half-time that is independent of the duration of infusion. Remifentanil's distinctive mode of esterase metabolism is unaffected by hepatic or renal dysfunction. The fentanyl congeners all have potential risks for bradycardia, hypotension, and skeletal muscle rigidity when administered rapidly in large doses. While the most advantageous regimen available appears to be a combination of a sedative-hypnotic and opioid agent, the synergistic potential for cardiopulmonary depression with such concurrent therapies needs to be stressed.

A relatively new drug possessing uniquely combined sedative and analgesic properties is dexmedetomidine. It is a selective alph-2 agonist designated for IV infusion administration. It provides anxiolysis, sedation, and analgesia, while maintaining hemodynamic stability and minimal respiratory depression (32). Dexmedetomidine exhibits a slow onset of effect and an elimination half-time of six hours (33).

\section{Monitoring}

Undertaking a sedation analgesic course of therapy in support of an interventional procedure requires that patients are monitored appropriately during the procedure (34-36). The most common adverse effects from sedation analgesia is excessive CNS depression and consequent ventilatory and hemodynamic failure. Monitoring parameters should include continuous noninvasive blood pressure, pulse respiratory rate, and pulse oximetry, and periodic assessments of sedation level $(37,38)$. Supplemental oxygen, suction apparatus, and emergency airway management equipment should be readily available. While monitoring physiologic parameters, be especially vigilant for the following: decreases in oxygen saturation for 30 seconds or longer of more than $10 \%$ or for adult and pediatric patients and $5 \%$ or more for patients age 65 or older; signs of respiratory distress or airway obstruction; decrease in blood pressure more than $10 \%$ below baseline; vasovagal reaction; or loss of consciousness. Interventions in response to any alterations in the monitored parameters should include verbal or physical stimulation of the patient, supplemental oxygen, and possible administration of a reversal agent, such as naloxone or flumazenil (39).
Guidelines

Practice guidelines for sedation and analgesia by non-anesthesiologists were established by the American Society of Anesthesiologists Task Force in 2001 (2). More recently, a formal declaration distinguishing monitored anesthesia care from moderate sedation analgesia was approved by the American Society of Anesthesiologists House of Delegates in 2004 (40).

\section{CONCLUSION}

Sedation encompasses a progressive continuum of stages ranging from relaxation and anxiolysis to general anesthesia. Recent trends in the development of sedation analgesic drugs have emphasized higher specificity of effect, greater controllability of action, and enhanced predictability of outcome. Drugs within a particular class are considered to share a common mechanism of action and produce a similar spectrum of effects. Tolerance to the effects of most sedative analgesic drugs develops with repeated, longterm use. Along with this tolerance phenomenon is the potential for physical dependence, that manifests as a withdrawal syndrome upon discontinuation of drug use. Side effects and complications associated with the sedation analgesic drugs usually result from a dose-dependent extension of their pharmacologic spectrum of effects, hypersensitivity reaction, or drug interaction. The pharmacologic properties and effects of sedation analgesic drugs are most clinically beneficial when applied in a manner that best utilizes their advantages appropriate to the specific requirements of a particular situation. Sedation analgesia therapy provided in support of an interventional procedure requires strict patient monitoring. The ASA has established practice guidelines for sedation and analgesia by nonanesthesiologists.

\section{Author AfFilation:}

James D. Colson, MD

Clinical Assistant Professor of

Anesthesiology

Attending Staff, Center for

Interventional Pain Medicine

University of Michigan

$1 \mathrm{H} 247 \mathrm{UH}$,

1500 E. Medical Center Drive

Ann Arbor, MI 48130

E-mail:colsonj@umich.edu 


\section{REFERENCES}

1. Delgado $M$, Gempeler F, Rodriguez N Analgo-sedation for diagnostic and therapeutic endoscopic or radiologic procedures in adults. Cochrane Database Syst Rev 2004. www.cochrane.org

2. The American Society of Anesthesiologists Task Force on Sedation and Analgesia by Non-Anesthesiologists - Gross JB, Bailey PL, Connis RT, Cote CJ, Davis FG, Epstein BS, Gilbertson L, Nickinovich DG, Zerwas JM, Zuccaro G. Practice guidelines for sedation and analgesia by non-anesthesiologists. Anesthesiology 2002; 96:1004 1017.

3. Frolich MA, Dennis DM, Shuster JA, Melker RJ. Precision and bias of target controlled propofol infusion for sedation. $\mathrm{Br} / \mathrm{An}$ aesth 2005; 94:434-437.

4. Atack JR. Anxioselective compounds acting at the $G A B A(A)$ receptor benzodiazepine binding site. Curr Drug Targets CNS Neurol Disord 2003; 2:213-232.

5. Mohler H, Richards JG. The benzodiazepine receptor: a pharmacological control element of brain function. Eur J Anaesthe siol Suppl 1988; 2:15-24.

6. Atcheson R, Lambert DG. Update on opioid receptors. Br J Anaesth 1994; 73:132-134.

7. Stein C. The control of pain in peripheral tissue by opioids. N Engl J Med 1995; 332: 1685-1690.

8. Shafer SL, Stanski DR. Improving the clinical utility of anesthetic drug pharmacokinetics. Anesthesiology 1992; 76:327-330.

9. Schuttler J, Schwilden H, Stoekel H. Pharmacokinetics as applied to total intravenous anaesthesia. Practical implications. Anaesthesia 1983; 38:S53-S56.

10. Stoelting RK. Pharmacology and Physiology in Anesthesia Practice. 3rd ed. Lippin cott-Raven, Philadelphia, 1999.

11. Reves JG, Fragen RJ, Vinik HR, Greenblatt DJ. Midazolam: Pharmacology and uses. Anesthesiology 1985; 62:310-324.

12. Morgan GE, Mikhail MS, Murray MJ. Clinical Anesthesia. 3rd ed. McGraw-Hill, New York, 2002.

13. Smith I, White PF, Nathanson M, Gouldson R. Propofol. An update on its clinical use. Anesthesiology 1994; 81:1005-1043.

14. Evers AS, Maze M. Anesthetic Pharmacology: Physiologic Principles and Clinical Practice. Churchill Livingstone, Philadelphia, 2004.

15. Bissonnette B, Swan H, Ravussin P, Un V. Neuroleptanesthesia: Current status. Can J Anaesth 1999; 46:154-168.

16. Reich DL, Silvay G. Ketamine: an update on the first twenty-five years of clinical ex- perience. Can J Anaesth 1989; 36:186-197.

17. White PF, Way WL, Trevor AJ. Ketamine its pharmacology and therapeutic uses. Anesthesiology 1982; 56:119-136.

18. Bailey JM. Context-sensitive half-times: What are they and how valuable are they in anesthesiology? Clin Pharmacokinet 2002; 41:793-799.

19. Hughes MA, Glass PS, Jacobs JR. Contextsensitive half-time in multicompartment pharmacokinetic models for intravenous anesthetic drugs. Anesthesiology 1992; 76:334-341.

20. Manchikanti L, Damron KS, Rivera J, McManus CD, Jackson SD, Barnhill RC, Martin JC. Evaluation of effect of sedation as a confounding factor in the diagnostic validity of lumbar facet joint pain: A prospective, randomized, double-blind, placebo-controlled evaluation. Pain Physician 2004; 7:407-410.

21. Fragen RJ. Drug Infusions in Anesthesiolo$g y, 2^{\text {nd }}$ Ed. Lippincott-Raven, Philadelphia, New York, 1996.

22. Janzen PR, Hall WJ, Hopkins PM. Setting targets for sedation with a target-controlled propofol infusion. Anaesthesia 2000; 55:666-669.

23. White M, Kenny GN. Intravenous propofol using a computerized infusion system. Anaesthesia 1990; 45:204-209.

24. Manninen PH, Chan AS, Papworth D. Conscious sedation for interventional neuroradiology: A comparison of midazolam and propofol infusion. Can J Anaesth 1997; 44:26-30.

25. Hasen KV, Samartzis D, Casas LA, Mustoe TA. An outcome study comparing intravenous sedation with midazolam/fentanyl (conscious sedation) versus propofol infusion (deep sedation) for aesthetic surgery. Plast Reconstr Surg 2003; 112:1683-1691.

26. Borgeat A, Wilder-Smith $\mathrm{OH}$, Suter PM. The non-hypnotic therapeutic applications of propofol. Anesthesiology 1994; 80:642656.

27. Ulmer BJ, Hansen JJ, Overley CA, Symms MR, Chadalawada V, Liangpunsakul S, Strahl E, Mendel AM, Rex DK. Propofol versus midazolam/fentanyl for outpatient colonoscopy: administration by nurses supervised by endoscopists. Clin Gastroenterol Hepatol 2003; 1:425-432.

28. Barbi E, Gerarduzzi T, Marchetti F, Neri E, Verucci E, Bruno I, Martelossi S, Zanazzo G, Sarti A, Ventura A.. Deep sedation with propofol by non-anesthesiologists: a prospective pediatric experience. Arch Pediatr Adolesc Med 2003; 157:1097-1103.

29. Frazee BW, Park RS, Lowery D, Baire M. Propofol for deep procedural sedation in the
ED. Am J Emerg Med 2005; 23:190-195

30. Godambe SA, Elliot V, Matheny D, Pershad J. Comparison of propofol/fentanyl versus ketamine/midazolam for brief orthopedic procedural sedation in a pediatric emergency department. Pediatrics 2003; 112: 116-123.

31. Muellejans B, Lopez A, Cross MH, Bonome C, Morrison L, Kirkham AJ. Remifentanil versus fentanyl for analgesia based sedation to provide patient comfort in the intensive care unit: a randomized, doubleblind controlled trial [ISRCTN43755713]. Crit Care 2004; 8:R1-R11.

32. Hall JE, Uhrich TD, Barney JA, Arain SR, Ebert TJ. Sedative, amnestic, and analgesic properties of small-dose dexmedetomidine infusions. Anesth Analg 2000; 90: 699-705.

33. Venn RM, Karol MD, Grounds RM. Pharma cokinetics of dexmedetomidine infusions for sedation of postoperative patients requiring intensive care. Br J Anaesth 2002; 88:669-675.

34. Pitetti RD, Singh S, Pierce MC. Safe and efficacious use of procedural sedation and analgesia by non-anesthesiologists in a pediatric emergency department. Arch Pediatr Adolesc Med 2003; 157:1090-1096.

35. Malviya S, Naughton NN, Tremper KK. Sedation and Analgesia for Diagnostic and Therapeutic Procedures. Humana Press, Totowa, 2003.

36. Bell JK, Laasch HU, Wilbraham L, England RE, Morris JA, Martin DF. Bispectral index monitoring for conscious sedation in intervention: better, safer, faster. Clin Radiol 2004; 59:1106-1113.

37. Bitar G, Mullis W, Jacobs W, Matthews D, Beasley M, Smith K, Watterson P, Getz S, Capizzi P, Eaves F zrd. Safety and efficacy of office-based surgery with monitored anesthesia care/sedation in 4778 consecutive plastic surgery procedures. Plast $R e$ constr Surg 2003; 111:150-158.

38. Innes G, Murphy M, Nijssen-Jordan C Ducharme J, Drummond A. Procedural sedation and analgesia in the emergency department. Canadian Consensus Guidelines. J Emerg Med 1999; 17:145-156.

39. UMHHC Policy 62-11-001 Guidelines for the Use of Sedation Analgesia for Diagnostic, Therapeutic and Minor Surgical Procedures. University of Michigan Health System, Ann Arbor, MI, 2004. www.med.umich.edu/

40. Distinguishing Monitored Anesthesia Care ("MAC") From Moderate Sedation/analgesia (Conscious Sedation): American Society of Anes thesiologists, 2004. www.asahq.org/ publicationsandServices/standards/ 